\title{
Physicochemical Properties and Structural Characterisation of Fatty Acids Derived from Jatropha curcas Oil Using Microwave Assisted Alkaline Hydrolysis
}

\author{
Nur'aini Raman Yusuf, ${ }^{1}$ Ruzaimah Nik Mohamad Kamil ${ }^{1 *}$ and Suzana Yusup ${ }^{2}$ \\ ${ }^{1}$ Fundamental and Applied Sciences Department, Universiti Teknologi PETRONAS, \\ 32610 Bandar Seri Iskandar, Perak, Malaysia \\ ${ }^{2}$ Chemical Engineering Department, Universiti Teknologi PETRONAS, \\ 32610 Bandar Seri Iskandar, Perak, Malaysia \\ *Corresponding author: ruzaimah_nikmkamil@petronas.com.my
}

Published online: 15 February 2017

To cite this article: Yusuf, N. R., Kamil, R. N. M. \& Yusup, S. (2017). Physicochemical properties and structural characterisation of fatty acids derived from Jatropha curcas oil using microwave assisted alkaline hydrolysis. J. Phys. Sci., 28(Supp. 1), 269-279, https://doi.org/10.21315/jps2017.28.s1.18

To link to this article: https://doi.org/10.21315/jps2017.28.s1.18

\begin{abstract}
Fatty acids are important preliminary materials in biochemical industrial process. Alkaline hydrolysis process is known to be the easiest and fastest way to achieve maximum fatty acids yield. In this study, impact of microwave irradiation assistance on structural characterisation of fatty acids produced from Jatropha curcas oil using alkaline hydrolysis applied in production of fatty acids was verified using nuclear magnetic resonance (NMR) analysis and compared with standard oleic acids. Other physical properties such as free fatty acid, viscosity, density, cloud point and flash point were studied. ${ }^{1} \mathrm{H}-\mathrm{NMR}$ and ${ }^{13} \mathrm{C}-\mathrm{NMR}$ spectra showed similar structure for both synthesised fatty acids and standard oleic acids with a significant difference in carbon and proton length. The physical properties are also in good agreement for both fatty acids. These findings emphasise that alkaline hydrolysis process under the assistance of microwave irradiation did not change the properties of fatty acids but enhanced the fatty acids production to about $97 \mathrm{wt} \%$ with shorter reaction time of $15 \mathrm{~min}$.
\end{abstract}

Keywords: Fatty acids, microwave assisted heating, ${ }^{1} \mathrm{H}-\mathrm{NMR},{ }^{13} \mathrm{C}-\mathrm{NMR}$, Jatropha curcas 


\section{INTRODUCTION}

Studies on the application of microwave technology to improve conventional processes are on the rise. According to Oghbaei et al., since 1980s, microwave irradiation has been progressively replacing the conventional heating method and broadening the toolbox of chemistry, physics and material science. ${ }^{1}$ Conventional heating is a way to produce fats and oil products such as bio-diesel and fatty acids. However, as mentioned by Olabemiwo et al., this method appeared to be inefficient and time consuming due to the fact that the conventional method transfers energy from the shell of reaction vessel into the core of reaction system media and thus, leading to a large amount of energy and contributing to slower process kinetic. ${ }^{2}$ As reported by Cristina and Paolo, microwave irradiation has become a worldwide popular heating method to substitute the conventional method because it is proven to be clean, convenient, and most importantly, it produces higher yields and can be applied in a shorter reaction time. ${ }^{3}$ In polar medium, microwave irradiation transfers energy from core to the shell and throughout the process, the sample itself will generate heat on its own through penetration of microwave irradiation. Therefore, the process is expected to shorten the reaction time due to transfer of heat directly to the reactant.

Jatropha curcas oil is one of the most important seed oils as it contains high percentage of free fatty acids, thus enabling high potential for fatty acid and biolubricant productions. Literature on Jatropha curcas oil utilisation is increasing by years as the potential of Jatropha oil is intensely explored. Lin et al. found that using microwave irradiation with ionic liquid as the catalyst could lead to the Jatropha bio-diesel with yield of $98.5 \mathrm{wt} . \%$ methyl esters at milder experiment condition. ${ }^{4}$ Melo-Junior et al. explored the pre-treatment of fatty acids under microwave irradiation to promote non-catalytic esterification of oleic acid under a relatively high temperature and yield up to $60 \%$ conversion in 60 min of reaction. ${ }^{5}$ Both studies emphasised that the fast heating rate in microwave irradiation was responsible for the result enhancement as compared to conventional heating. They show that under the microwave irradiation, the pre-treatment may be considered as an effective and feasible method for sample production.

The common fatty acids present in many vegetable oils are mainly oleic, linoleic, and linolenic acids. Inekwe et al. found that the fatty acid structure is the major factor that influences their chemical and physical properties and strongly affects the use of oils as bio-lubricants and bio-diesel. ${ }^{6}$ There are various methods in determining these fatty acids such as gas chromatography and high performance liquid chromatography. The common method is gas chromatography but the samples have to be converted to corresponding methyl esters prior to the analysis. 
Besides, the spectroscopic methods are useful for the sample confirmation as it is not heat sensitive. As studied by Knothe and Kenar, these fatty acids can be quantified by using ${ }^{1} \mathrm{H}$-nuclear magnetic resonance spectroscopy ( $\left.{ }^{1} \mathrm{H}-\mathrm{NMR}\right) .{ }^{7}$

Despite previous research on application of microwave irradiation in bio-fuel and bio-refineries area, the use of microwave irradiation in an alkaline hydrolysis process for the fatty acid production has not yet been extensively addressed. In this study, fatty acid synthesis from alkaline hydrolysis via microwave irradiation was conducted and comparative structural analysis with commercial fatty acids was investigated. Furthermore, the comparative studies with respect to their physiochemical characteristics were highlighted.

\section{EXPERIMENTAL}

\subsection{Materials}

Jatropha curcas oil was used as feedstock and was purchased from Bionas Sdn. Bhd. Malaysia. Standard oleic acids were purchased from Sigma Aldrich (US) whilst commercial fatty acids from Glycerin Traders, LaPorte, US. Catalysts such as potassium hydroxide $(\mathrm{KOH})$, phenolphthalein and sodium hydroxide were purchased from Merck (Germany). In addition, solvents such as isopropanol, n-hexane, ethanol, dried methanol, and toluene were obtained from Fisher Chemicals (UK).

\subsection{Apparatus and Characterisation}

Experiments were conducted in a microwave synthesis MARS 6(CEM Corporation) with a working frequency of $2450 \mathrm{MHz}$ and an output power level set at maximum setting of $1800 \mathrm{~W}$ and was performed in an open vessel fitted with a condensing system. Validation of fatty acids chemical structure was confirmed using ${ }^{1} \mathrm{H}$ NMR and ${ }^{13} \mathrm{C}$ NMR spectroscopy. All spectra were recorded using NMR Bruker AVANCE $400 \mathrm{MHz}$ III operating at $400.17 \mathrm{MHz}$. The physical characterisations of Jatropha fatty acids for verification was analysed by acid value titration (AOCS Cd 3d-63) method, viscosity test using an Anton Paar DMA 5000 viscometer (ASTM D445), cloud and pour point measured by using CPP 5G'S following the ASTM D 2500 and ASTM D 97 while flash point analyser was done using a close-cup analyser (ISL, model) according to the test method ASTM D93 A, where liquid sample was heated under constant stirring at a steady rate of $5^{\circ} \mathrm{C} / \mathrm{min}-6^{\circ} \mathrm{C} / \mathrm{min}$ and the flash point was determined using an igniter at specified temperature intervals. 


\subsection{Microwave Assisted Experiment}

The alkaline hydrolysis procedure was carried out according to Salimon et al. with some modifications in microwave irradation process. ${ }^{8}$ The experiment for this research work was performed totally in the microwave equipment, in contrast to Salimon et al. ${ }^{8}$ A solution containing Jatropha curcas oil and ethanolic $\mathrm{KOH}$ was mixed in a $500 \mathrm{~mL}$ reaction flask and reacted in a microwave synthesis reactor by setting the optimum parameters. ${ }^{9}$ The optimum parameters are temperature at $65^{\circ} \mathrm{C}$, reaction time of $15 \mathrm{~min}$, oil to solvent ratio of $1: 68,1.75 \mathrm{M}$ concentration of ethanolic $\mathrm{KOH}$. The reaction mixture was then acidified with $6 \mathrm{~N}$ hydrocloric acid and then followed by a separation proces with hexane in order to recover the fatty acids. The fatty acids was then thoroughly washed with distilled water until neutral $\mathrm{pH}$ and dried with an anhydrous magnesium sulphate. The solvent was evaporated in a rotary vacuum evaporator to recover the fatty acids.

\section{RESULTS AND DISCUSSION}

\subsection{Microwave-assisted for Alkaline Hydrolysis Process}

The alkaline hydrolysis process have successfully converted Jatropha curcas oil to fatty acids via microwave irradiation technique. The reaction scheme of this process is shown in Figure 1. The optimum parameters were selected based on our previous research work. ${ }^{9}$ Roberts and Strauss mentioned that an enhancement of reaction in microwave heating is achieved due to its circular motion wave that generates a complete heat transfer to the reactants. ${ }^{10}$ Hence, this induced the real reaction temperature higher than the average temperature of medium.

As described by Sajjadi et al., two movements are involved in the mechanism of base-catalysed ester hydrolysis. ${ }^{11}$ They consist of dipolar rotation (from polar group such as alcohol, triglyceride, etc.) and ionic conduction (from catalyst molecules such as $\mathrm{K}^{+} \mathrm{OH}^{-}$). These movements are significant in microwave irradiation as the efficiency of the reaction is based on the ability of liquids and solids to absorb and transform electromagnetic energy into heat. Given that the microwave is applied to reaction mixtures, the first movement involves dipole molecules continuously aligning themselves to an electric field. Subsequently, the molecule orientation will result in increase in friction and kinetic energy. The second movement is effected as the charge of molecule exposed to the electric field, resulting in the movement of ions back and forth through the sample, therefore generating the heat. 


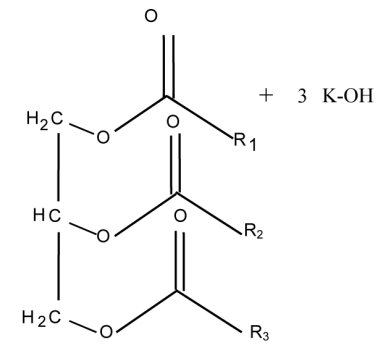

Triglyceride

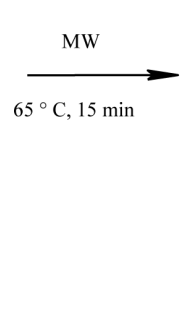

$\mathrm{R}_{1}=\left(\mathrm{CH}_{2}-\mathrm{CH}_{2}\right) \mathrm{n}-\mathrm{CH}_{3}$,

$\mathrm{R}_{2}=\mathrm{CH}_{2}=\mathrm{CH}-\left(\mathrm{CH}_{2}\right) \mathrm{n}-\mathrm{CH}_{3}$,

$\mathrm{R}_{3}=\mathrm{CH}_{2}=\mathrm{CH}-\left(\mathrm{CH}_{2}\right) \mathrm{n}-\mathrm{CH}=\mathrm{CH}-\left(\mathrm{CH}_{2}\right) \mathrm{n}-\mathrm{CH}_{3}$
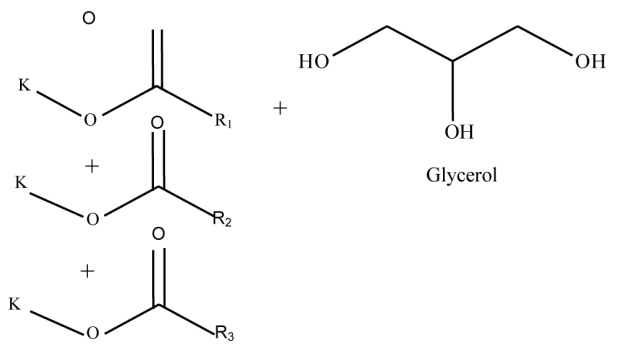

Fatty Acids Potassium

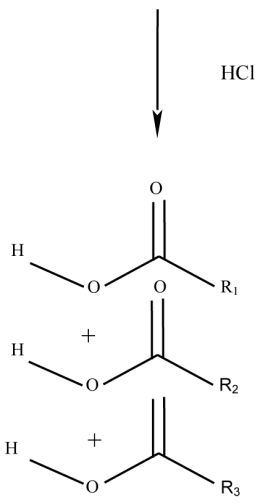

Fatty Acids
$3 \mathrm{KCl}$

Salt

Figure 1: Reaction scheme of alkaline hydrolysis of Jatropha curcas oil. ${ }^{8}$

The effect of this molecular movement during the hydrolysis of ester from triglyceride (Jatropha curcas oil) chain is presented in Figure 2. The increase of the heat transfer in the system mainly influences the rate of ester chain breakage of the and thus creates an intermediate tetrahedral. This unstable intermediate reforms the carboxylic acid in the second step by an elimination of alkoxide group. Consequently, the alkoxide group takes place as a base to deprotonate the carboxylic acid. The carboxylic acid group is obtained through acidification with hydrochloric acid.

\subsection{Physical Properties of SynthesiSed and Commercial Fatty Acids}

The appearance of synthesised fatty acids from Jatropha curcas oil is yellowish while the commercial fatty acid is brown in colour. Physical properties of synthesised fatty acids of Jatropha curcas oil and comparison with commercial 

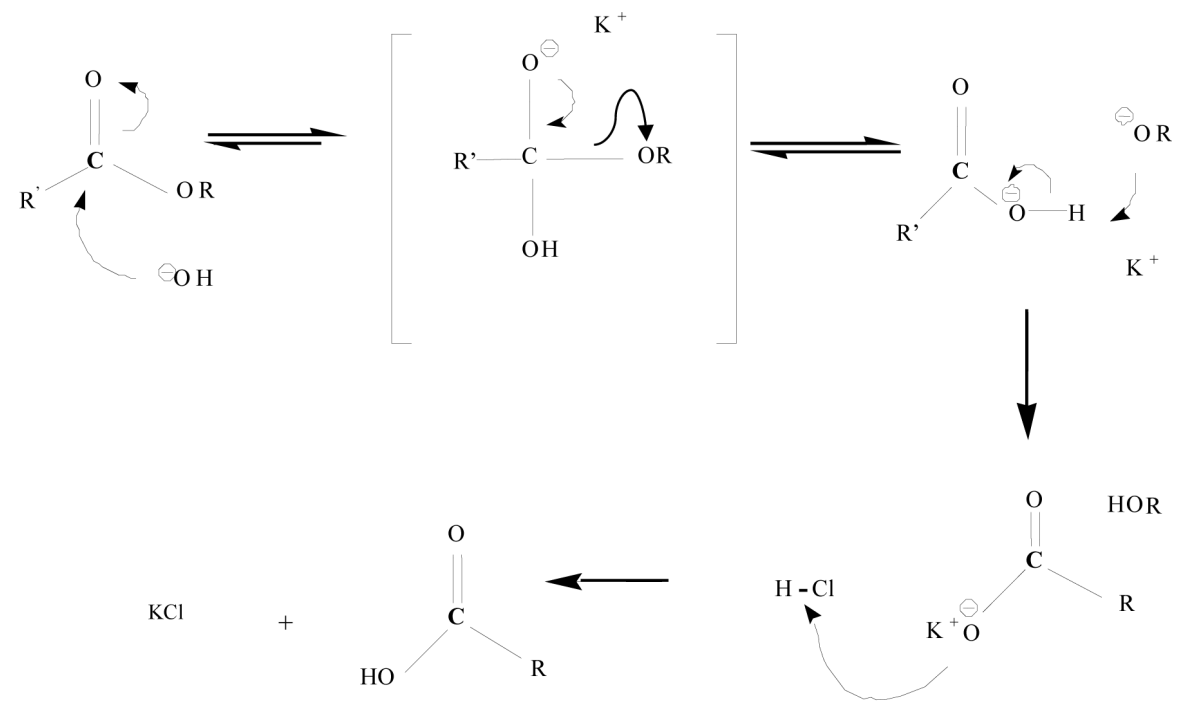

$\mathrm{R}^{\prime}=\mathrm{R}_{1}, \mathrm{R}_{2}$ and $\mathrm{R}_{3}$

Figure 2: Mechanism alkaline hydrolysis of Jatropha curcas oil. ${ }^{10}$

fatty acids are shown in Table 1. As shown in Table 1, almost $97 \mathrm{wt} . \%$ fatty acids are synthesised from alkaline hydrolysis through the microwave irradiation process. This value is substantially higher when compared to the commercial fatty acid that has been synthesised via a conventional approach and different precursors. Therefore, it highlights the successful conversion of triglyceride to fatty acids when applying the microwave technique and it can be justified by a faster rate of ester bond-breaking resulted from an exposure of the molecular charge towards the electric field.

Table 1: Comparison physical properties between synthesised and commercial fatty acids.

\begin{tabular}{lccc}
\hline \multirow{2}{*}{ Properties } & \multirow{2}{*}{ Units } & \multicolumn{2}{c}{ Fatty Acids } \\
\cline { 3 - 4 } & & This work & Commercial \\
\hline Free fatty acids & $\mathrm{wt} \%$ & 97 & 99 \\
Viscosity @ $40^{\circ} \mathrm{C}$ & $\mathrm{mPa} \cdot \mathrm{s}$ & 16.79 & 20 \\
Density @ $40^{\circ} \mathrm{C}$ & $\mathrm{g} / \mathrm{cm}^{3}$ & 0.88 & 0.91 \\
Cloud point & ${ }^{\circ} \mathrm{C}$ & -5.1 & 14 \\
Pour point & ${ }^{\circ} \mathrm{C}$ & 12 & 10 \\
Flash point & ${ }^{\circ} \mathrm{C}$ & 204 & 190 \\
\hline
\end{tabular}


According to Diamante and Lan, liquid viscosity is amongst the important characteristics because it will determine the quality of the fatty acids. ${ }^{12}$ In Table 1 , it can be seen that the viscosity of synthesised fatty acids is $16.79 \mathrm{mPa} . \mathrm{s}$ and this value is in agreement with the data range reported by Noureddini et al. $(17.7 \mathrm{mPa} \cdot \mathrm{s}){ }^{13}$ Nevertheless, the viscosity of commerial fatty acids shows a slightly higher value as compared to the synthesised fatty acids in this study. The low viscosity obtained when applying the microwave irradiation processing may be attributed to a better absorption rate of polar solvent and lipids, and thus, resulting in an upgrading in reaction kinetics.

According to Esteban et al., if the relationship between density and viscosity is known then one only needs to measure the density of the respected oil to deduce its viscosity value. ${ }^{14}$ In this work, the values of viscosity and density of the synthesised fatty acids and commercial fatty acids are slightly different, and this scenario may be due to the difference in molecular chain length. Accordingly, the high value of commercial fatty acids implies that the carboxylic chain is extensively lengthy as compared to the synthesised fatty acids.

The cloud and pour point are significant parameters that can indicate the suitability of the particular fuel for low temperature applications. As reported by Reaume and Ellis, these properties will indicate the tendency of oil to plug the filter at cold operating temperature. ${ }^{15}$ The cloud point of synthesised fatty acids that is around negative $5.1^{\circ} \mathrm{C}$ signifies that it can be applied in cold operating condition, in contrary to the commercial fatty acids. Meanwhile, the pour point of synthesised fatty acids is $12^{\circ} \mathrm{C}$ which is slightly higher as compared to the commercial fatty acids. ${ }^{16}$ Both criterias indicate that the synthesised fatty acids can be used in low temperature applications. Nevertheless, criteria for the commercial fatty acids were chosen by end users depending on the targeted application.

Besides, flash point which is the minimum temperature for the sample to self-ignite is significant as it determines the safety during the transport, storage and handling. The flash point of the synthesised fatty acids that is around $204^{\circ} \mathrm{C}$ implies that it can be safely utilised at higher temperature conditions.

\subsection{NMR Analysis}

\subsubsection{H-NMR spectra of synthesised fatty acids and standard oleic acids}

The verification of the fatty acid structures was confirmed by ${ }^{1} \mathrm{H}-\mathrm{NMR}$ analysis in Figure 3, which shows a comparison in NMR spectra for the synthesised fatty acids with the standard oleic acids. Referring to Figure 3, the identical ${ }^{1} \mathrm{H}-\mathrm{NMR}$ 
spectra of synthesised fatty acids to standard oleic acids ascertains the high purity of the synthesised fatty acids. Figure 3 shows the characteristic signals at $0.8-0.9$ ppm for methyl $\left(-\mathrm{CH}_{3}\right)$ group proton, 1.0 to $1.8 \mathrm{ppm}$ signal for methylene $\left(-\mathrm{CH}_{2}\right)$ proton, 2.0 to $2.1 \mathrm{ppm}$ signal for proton attached to allylic carbons corresponding to the Salimon et al. ${ }^{8}$ The allylic carbon arose from carbon attached to carbon double bonds $(-\mathrm{H}-\mathrm{C}-\mathrm{C}=\mathrm{CH})$ whereas the peak at $5.8 \mathrm{ppm}$ arose from proton attached to carbon double bonds $(\mathrm{HC}=\mathrm{CH})$. Broad peak at 10 to $12.1 \mathrm{ppm}$ corresponds to carboxyl groups as reported by Barison et al. ${ }^{17}$

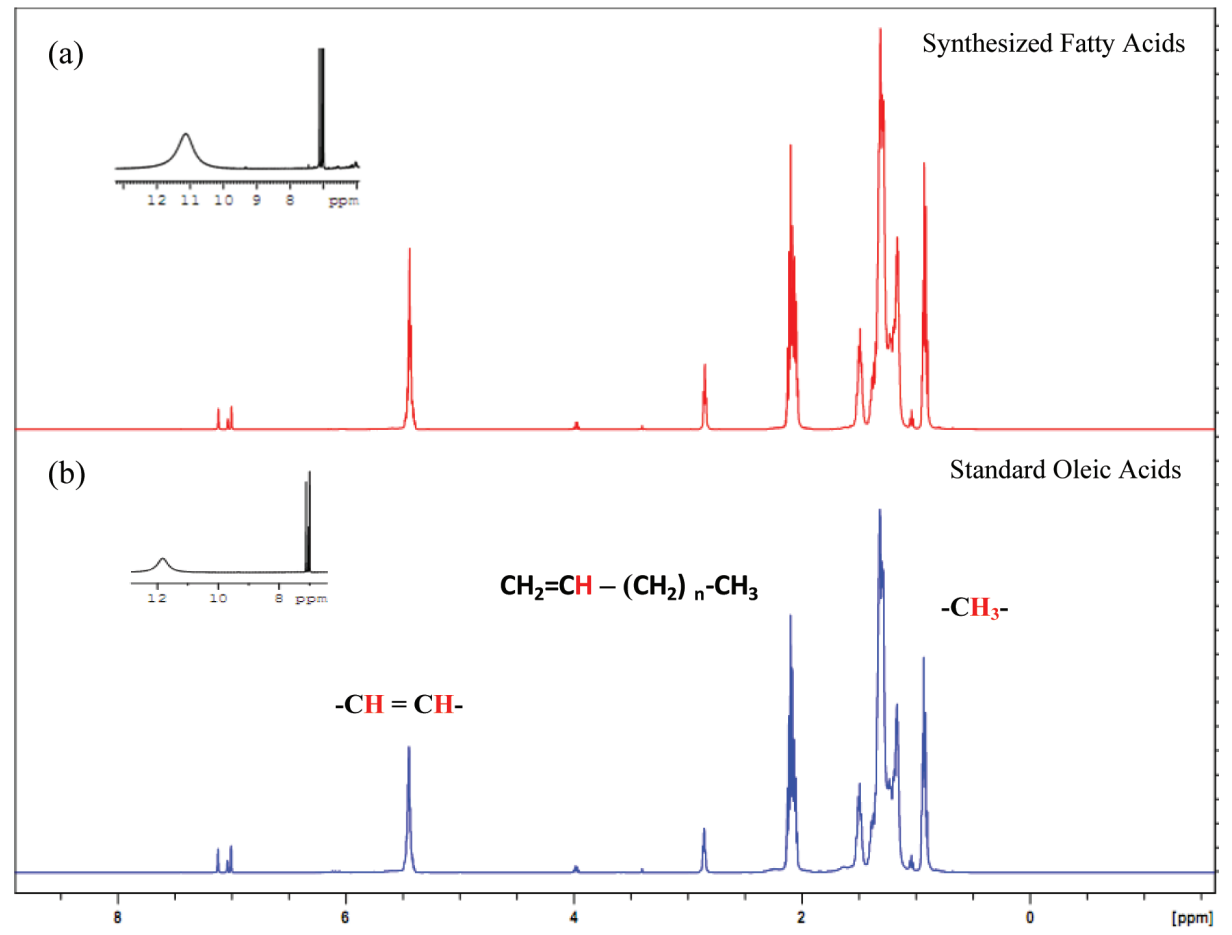

Figure 3: Comparison of proton spectra of fatty acids between (a) synthesised and (b) standard oleic acids.

\subsection{2 ${ }^{13} \mathrm{C}-\mathrm{NMR}$ spectra of synthesised fatty acids and standard oleic acids}

Previous research work by Amir et al. reported that determination of fatty acids could be done based on the proton decoupling in ${ }^{13} \mathrm{C}-\mathrm{NMR} .{ }^{18}$ The proton decoupling consists of a set of single peak, wherein each carbon is attached to proton. Figure 4 displays the comparison in the carbon spectra between the synthesised and commercial fatty acids. The spectra consist of the alkane carbon $\left(\mathrm{CH}_{3}\right)$ and then allylic and divinyl carbons (carbon attach to double bond carbon) at 20 to $40 \mathrm{ppm}$. 
Nevertheless, in spectrum (b), there are slightly difference in the peak number and it shows an additional alkane carbon. Similar phenomena occurs in spectrum (b) whereby an appearance of $\mathrm{C}=\mathrm{C}$ at 120 to $140 \mathrm{ppm}$ shows the additional double bond of carbon. On the other hand, the carbon for carboxylic acids $\left(\mathrm{R}-\mathrm{CO}_{2} \mathrm{H}\right)$ appears at $180 \mathrm{ppm}$.

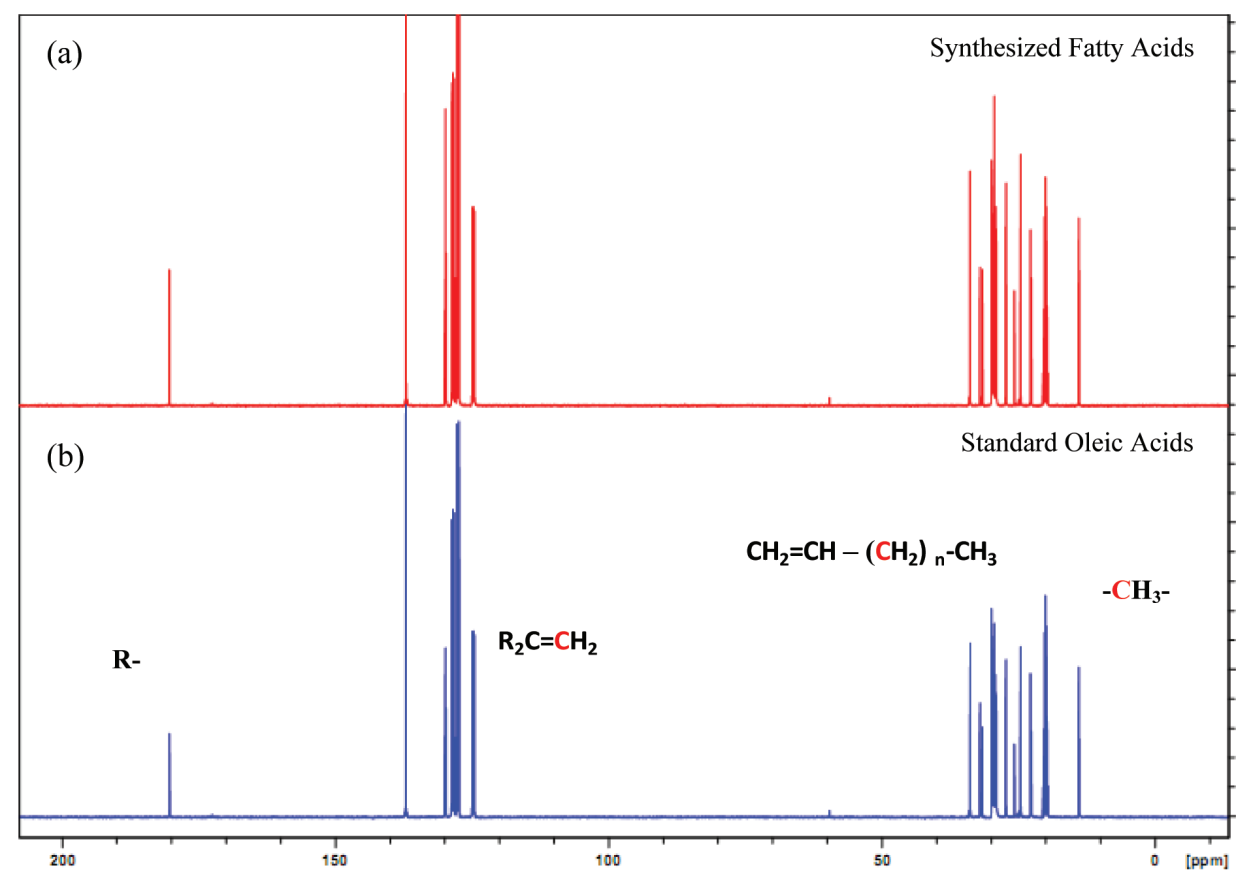

Figure 4: Comparison of ${ }^{13} \mathrm{C}-\mathrm{NMR}$ carbon spectra of fatty acids between (a) synthesised and (b) standard oleic acids.

\section{CONCLUSION}

Fatty acids derived from alkaline hydrolysis via microwave irradiation technique was successfully synthesised in research study. The physicochemical characterisation was compared to commercial fatty acids to identify the conversion and quality of the fatty acids. Based on the physiochemical characteristics, it was proven that the synthesised fatty acids are equivalent to commecial fatty acids, except a slight difference in the chain length. The capability of microwave irradiation in alkaline hydrolysis may be due to better absorption rate of solvent and lipid, since both of them are polar in nature. Besides, the NMR analysis acknowledged identical composition between the synthesised fatty acids and standard oleic acids. 


\section{ACKNOWLEDGEMENTS}

Authors gratefully acknowledge financial supports from the Ministry of Higher Education Malaysia (MOHE) for the LRGS, Universiti Teknologi PETRONAS for STIRF Grant and MyBrain 15. Authors also would like to thank to laboratory technical assistants and others in the Biomass Processing Laboratory for their assistance.

\section{REFERENCES}

1. Oghbaei, M. \& Mirzaee, O. (2010). Microwave versus conventional sintering: A review of fundamentals advantages and applications. J. Alloys Comp., 494, 175-189, https://doi.org/10.1016/j.jallcom.2010.01.068.

2. Olabemiwo, O. M. et al. (2014). Effect of microwave heating on fatty acid profiles of three nigerian vegetable oils. J. Appl. Chem., 7, 51-54.

3. Cristina, L. \& Paolo, V. (2015). Microwave reactors for chemicals synthesis and biofuels preparation. In Fang, Z. et al. (Eds). Biofuels and bio refineries, 17-40. London: Springer.

4. Lin, Y. C. et al. (2015). Energy-saving and rapid transesterification of Jatropha oil using a microwave heating system with ionic liquid catalyst. J. Taiwan Inst. Chem .Eng, 49, 72-78, https://doi.org/10.1016/j.jtice.2014. 11.014 .

5. Melo-Júnior, C. A. et al. (2009). Use of microwave irradiation in the noncatalytic esterification of c18 fatty acids. Energy Fuels, 23, 580-585, https://doi.org/10.1021/ef800766x.

6. Inekwe, U. V. et al. (2012). Fatty acid composition and physicochemical properties of Jatropha curcas oils from Edo and Kaduna states of Nigeria and India. Ann. Biol. Res., 3, 4860-4864.

7. Knothe, G. \& Kenar, J. A. (2004). Determination of the fatty acid profile by ${ }^{1}$ HNMR spectroscopy. Eur. J. Lipid Sci. Tech., 2, 88-96, https://doi. org/10.1002/ejlt.200300880.

8. Salimon, J., Abdullah, B. M. \& Salih, N. (2011). Hydrolysis optimisation and characterization study of preparing fatty acids from Jatropha curcas seed oil. Chem. Cent. J., 5, 1-9, https://doi.org/10.1186/1752-153X-5-67.

9. Yusuf, N. R., Kamil R. N. M. \& Yusup, S. (2015). Response surface methodology approach for optimisation of alkaline hydrolysis of Jatropha curcas oil via microwave assisted. Chem. Eng. Transact., 45, 1513-1518.

10. Roberts, B. A. \& Strauss C. R. (2005). Toward rapid green predictable microwave-assisted synthesis. Acc. Chem. Res., 38, 653-661, https://doi.org/ $10.1021 /$ ar040278m. 
11. Sajjadi, B., Aziz, A. A. \& Ibrahim, S. (2014). Investigation, modelling and reviewing the effective parameters in microwave-assisted transesterification. Renew. Sust. Energy Rev., 37, 762-777, https://doi.org/10.1016/j.rser.2014. 05.021 .

12. Diamante, L. M. \& Lan, T. (2014). Absolute viscosities of vegetable oils at different temperatures and shear rate range of 64.5 to $4835 \mathrm{~s}^{-1}$. J. Food Process., Article ID 23458.

13. Noureddini, H., Teoh, B. C. \& Clements, L. D. (1992). Viscosities of vegetable oils and fatty acids. J. Am. Oil Chem. Soc., 12, 1189-1191, https://doi.org/10.1007/BF02637678.

14. Esteban, B. et al. (2012). Temperature dependence of density and viscosity of vegetable oils. Biomass Bioenergy, 42, 164-171, https://doi.org/10.1016/j. biombioe.2012.03.007.

15. Reaume, S. J. \& Ellis, N. (2013). Use of isomerization and hydroisomerization reactions to improve the cold flow properties of vegetable oil based biodiesel. Energies, 2, 619-633, https://doi.org/10.3390/ en6020619.

16. Glycerin Traders. (2010). Material data safety sheet: Fatty acids. Indiana: Glycerin Traders.

17. Barison, A. et al. (2010). A simple methodology for the determination of fatty acid composition in edible oils through 1H NMR spectroscopy. Magn. Reson. Chem., 8, 642-650, https://doi.org/10.1002/mrc.2629.

18. Amir, S. et al. (2004). Elemental analysis, FTIR and 13C-NMR of humic acids from sewage sludge composting. Agronomie, 1, 13-18, https://doi. org/10.1051/agro:2003054. 
